\title{
Association of different enteroviruses with atopy and allergic diseases in early childhood
}

\author{
Tiina Palmu ${ }^{1,2}$ @ | Jussi Lehtonen ${ }^{1}$ | Laura Korhonen ${ }^{1,3}$ | Suvi M. Virtanen ${ }^{4,5,6,7}$ \\ Onni Niemelä8 $^{8}$ | Jorma Toppari, ${ }^{9}, 10$ | Jorma llonen ${ }^{11}$ | Riitta Veijola ${ }^{12}$ | \\ Mikael Knip ${ }^{13,14,15}$ @ | Olli H. Laitinen ${ }^{1}$ | Maria Lönnrot ${ }^{1,3}$ | Heikki Hyöty ${ }^{1,16}$ \\ ${ }^{1}$ Department of Virology, Faculty of Medicine and Health Technology, Tampere University, Tampere, Finland \\ ${ }^{2}$ Department of Dermatology and Allergology, The Hospital District of South Ostrobothnia, Seinäjoki, Finland \\ ${ }^{3}$ Department of Dermatology, Tampere University Hospital, Tampere, Finland \\ ${ }^{4}$ Health and Well-Being Promotion Unit, Finnish Institute for Health and Welfare, Helsinki, Finland \\ ${ }^{5}$ Unit of Health Sciences, Faculty of Social Sciences, Tampere University, Tampere, Finland \\ ${ }^{6}$ Tampere University Hospital, Research, Development and Innovation Center, Tampere, Finland \\ ${ }^{7}$ Center for Child Health Research, Tampere University and Tampere University Hospital, Tampere, Finland \\ ${ }^{8}$ Department of Laboratory Medicine and Medical Research Unit, Seinäjoki Central Hospital and Tampere University, Seinäjoki/Tampere, Finland \\ ${ }^{9}$ Institute of Biomedicine, Centre for Integrative Physiology and Pharmacology, and Centre for Population Health Research, University of Turku, Turku, Finland \\ ${ }^{10}$ Department of Pediatrics, Turku University Hospital, Turku, Finland \\ ${ }^{11}$ Immunogenetics Laboratory, Institute of Biomedicine, University of Turku, Turku, Finland \\ ${ }^{12}$ Department of Pediatrics, University of Oulu and Oulu University Hospital, Oulu, Finland \\ ${ }^{13}$ Pediatric Research Center, Children's Hospital, University of Helsinki and Helsinki University Hospital, Helsinki, Finland \\ ${ }^{14}$ Research Program for Clinical and Molecular Metabolism, Faculty of Medicine, University of Helsinki, Helsinki, Finland \\ ${ }^{15}$ Department of Pediatrics, Tampere University Hospital, Tampere, Finland \\ ${ }^{16}$ Fimlab Laboratories, Pirkanmaa Hospital District, Tampere, Finland
}

Correspondence

Tiina Palmu, Hanneksenrinne 7, 60220

Seinäjoki, Finland.

Email: tiina.palmu@tuni.fi

\section{Funding information}

This study was supported by funding from the Finnish Society of Allergology and Immunology, the Ida Montin Foundation, the Finnish Dermatological Society, the Finnish Medical Foundation, the Tampere Tuberculosis Foundation, the Päivikki and Sakari Sohlberg Foundation, the Academy of Finland (Prevaller Consortium), the Juho Vainio Foundation, the Yrjö Jahnsson Foundation, the Competitive Research Funding of the Tampere University Hospital (Grant Nos. 9L035, 9M029, 9P017, 9P057, 9R012, 9R055, 9S015, 9S074, 9T072, 9U016, 9U065, and 9V012), and the Medical Research Funds of Turku and Oulu University Hospitals.

Editor: Carmen Riggioni

\begin{abstract}
Background: Enterovirus (EV) infections, being among the most prevalent viruses worldwide, have been associated with reduced risk of allergic diseases. We sought to determine the association between EVs and allergic sensitization and disease in early childhood.

Methods: The study was carried out in a nested case-control setting within a prospective birth cohort in Finland. We included 138 case children who had specific IgE (s-IgE) sensitization at the age of 5 years and 138 control children without s-lgE sensitization. Allergic disease was recorded at study visits and identified with the ISAAC questionnaire. We screened for the presence of serotype-specific antibodies against $41 \mathrm{EVs}$ at 1-5 years of age and assessed their association with allergic sensitization and disease.

Results: The overall number of EV infections did not differ between s-lgE-sensitized children and non-sensitized control children. However, there was a tendency of case children with an allergic disease having less EV infections than their controls. This
\end{abstract}

This is an open access article under the terms of the Creative Commons Attribution NonCommercial License, which permits use, distribution and reproduction in any medium, provided the original work is properly cited and is not used for commercial purposes.

(c) 2021 The Authors. Pediatric Allergy and Immunology published by European Academy of Allergy and Clinical Immunology and John Wiley \& Sons Ltd. 
observation was statistically significant for species A EVs in case children with atopic dermatitis vs. control children: OR 0.6 (95\% Cl 0.36-0.99), $p=.048$.

Conclusion: This study supports the evidence that EV exposure and development of allergic disease are inversely associated. Interestingly, the inverse association was not observed for bare atopic IgE sensitization, but for IgE sensitization coupled with clinical atopic disease. This suggests that environmental factors influencing IgE sensitization may differ from those influencing progression to clinical allergic disease.

\section{KEYWORDS}

allergic rhinitis, allergy, asthma, atopic dermatitis, atopic sensitization, children, enterovirus

\section{1 | INTRODUCTION}

Atopy is defined as a tendency to produce IgE antibodies in response to ordinary harmless exposures to allergens. The prevalence of atopic IgE sensitization in developed countries is extremely high, rising from around $12 \%$ at the age of 1 year to around $30 \%$ at the age of 6 years ${ }^{1}$ and staying at around $20 \%-$ $50 \%$ throughout adulthood. ${ }^{2}$ The rapid increase in the prevalence during the last half a century ${ }^{3}$ suggests an involvement of environmental factors.

According to the hygiene/biodiversity hypothesis, ${ }^{4,5}$ the increase in atopy and allergic diseases is caused by a reduction in microbial exposures in childhood and, thus, skewing of the immune responses toward allergy-prone Th2-based reactions. Viruses guide the immune system toward Th1 direction. ${ }^{6}$ One could thus hypothesize that virus infections reduce the risk of atopy and allergy. Accordingly, low-grade inflammation, induced, for example, by microbial exposure during infancy, has been shown to decrease allergen-specific $\operatorname{lgE}(\mathrm{s}-\lg \mathrm{E})$ sensitization. ${ }^{7}$ Multiple viruses have been linked to the reduction of allergic diseases and/or IgE sensitization, ${ }^{8-13}$ but there are also reports contradicting the role of viruses. $3,9,14,15$

Enterovirus infections (EVs) are among the most common human virus infections worldwide. The classic human EVs can be classified into four species (EV-A, EV-B, EV-C, and EV-D) comprising more than 100 serotypes. The pathophysiology of different serotypes differs depending on their tropisms to different tissues and cells. Enterovirus infections are mostly asymptomatic or mild such as common cold or hand, foot, and mouth disease, but also serious diseases such as meningitis, myocarditis, and paralysis may occur. ${ }^{16}$ In in vitro models, EVs have been shown to bias the immune reactions toward the mature Th1-like pathway. ${ }^{17,18}$ They have also been linked with reduced risk of allergic diseases/atopy, ${ }^{11,12,19}$ but the results are contradictory. ${ }^{20,21}$

We contribute new insight into the association between EVs and atopy with respect to the individual EV serotypes. This study is the most comprehensive of its kind, containing 41 different human EV serotypes analyzed in a large case-control setting.

\section{Key Message}

Hygiene hypothesis has long been proposed to explain the increasing incidence of allergic sensitization and disease. Enteroviruses, the most common viruses infecting human beings, have been associated with the risk of allergy and asthma, but it is not known whether this association links to certain enterovirus types. The present study utilized the most comprehensive serotype panel thus far applied and was able to strengthen previous indications of a possible role of enteroviruses in the regulation of the risk of allergic diseases.

\section{2 | METHODS}

\subsection{Study population}

The study population was derived from the prospective populationbased Type 1 Diabetes Prediction and Prevention (DIPP) birthcohort study described in detail previously. ${ }^{22}$ Briefly, children born in the Tampere, Turku, and Oulu University Hospitals in Finland are screened at birth for HLA genotypes conferring to increased risk of type 1 diabetes. Written informed consent was obtained from caretakers both for HLA screening and for follow-up. The study protocol was approved by the ethics committees of the participating hospitals. The present study included children born between years 1995 and 2004 in Tampere and Oulu where children carrying the HLADQB1 genotypes ${ }^{*} 02 /{ }^{*} 0302$ and ${ }^{*} 0302 / x(x \neq * 2),{ }^{*} 0301$, or ${ }^{*} 0602$ were invited to follow-up. Blood samples were drawn at 3, 6, 12, 18, and 24 months of age and once a year thereafter. Atopic case and non-atopic control subjects were identified among children with an available serum sample for both EV antibody assay (serum sample between 1 and 5 years of age) and s-IgE antibody assay (serum sample at 5 years of age). Altogether, 138 case children with a positive $\mathrm{s}-\lg \mathrm{E}$ test and 138 matched control children with a negative s-IgE test were identified. Of the 138 case children, 65\% were sensitized 
to the food allergen mixture and $71 \%$ to the inhalant allergen mixture. Of those positive for foods, $57 \%$ tested positive for cow's milk, $32 \%$ for egg, $22 \%$ for wheat, and $6 \%$ for fish. Of those positive for inhalants, $57 \%$ were positive for birch, $37 \%$ for timothy, 3\% for cat, and $10 \%$ for house dust mite. The pairs were matched by gender and age at the EV antibody measurement ( \pm 3 months). The proportion of boys was $65 \%$.

Allergic diseases were identified at 5 years of age by the ISAAC questionnaire questions: "Has your child ever had asthma/hay fever or some other allergic rhinitis/atopic eczema diagnosed by a doctor?" The ISAAC data were missing in 39 children. Their allergic disease diagnoses were retrieved from the DIPP database: atopic dermatitis (ICD-code L20.x), asthma (J45.x), and allergic rhinitis/hay fever (J30.x) reported and confirmed by a physician by 5 years of age. Sixty-four (46\%) out of the 138 IgE-sensitized case children reported an allergic disease (47 atopic dermatitis, 27 allergic rhinitis, and 15 asthma) and formed a case subgroup for analysis of enteroviruses' association with clinical disease. These 64 case children were compared with their IgE-negative matched control children. Some of the 64 IgE-negative control children also reported an allergic disease ( $n=17$ ), but were eligible as controls, as IgE sensitization was the feature defining case vs. control status. Thirty-one (22.5\%) out of the total of 138 IgE-negative control children reported an atopic disease ( 25 atopic dermatitis, 2 allergic rhinitis, and 8 asthma reports).

Background data of the participants retrieved from the DIPP database included information of the age at the entry of day care, duration of breastfeeding, level of maternal and paternal education, HLA-DQB1 genotype, the presence of type 1 diabetes-associated islet autoantibodies, pets at home, older siblings, and maternal smoking during pregnancy. Parental atopy background information was available in the ISAAC data for all but 39 children. Maternal smoking was excluded from the analyses as it was reported by only five mothers.

\section{2 | Testing for allergen-specific lgE}

The s-IgE antibodies were tested at the age of 5 years against a mixture of airborne and food allergens (a multi-allergen test Phadiatop ${ }^{\circledR}$; Thermo Fisher Scientific, Uppsala, Sweden) using the ImmunoCAP ${ }^{\circledR}$ enzyme immunoassay (Thermo Fisher Scientific, Uppsala, Sweden) according to the manufacturer's protocol. The test was regarded positive if the food or inhalant allergen mixture showed a result of $0.35 \mathrm{kU} / \mathrm{L}$ or more. Individual s-IgE results were available for cow's milk, hen's egg, fish, wheat, house dust mite, cat, birch, and timothy grass.

\section{3 | Enterovirus seroneutralization assays}

Neutralizing antibodies were measured in serum or plasma against 41 EV serotypes listed in Table 2 as previously described. ${ }^{23}$ Viruses were isolated and cultivated, and seroneutralization assays were performed using the A549, Vero, and RD (all bought from American
Type Culture Collection) and GMK (from the National Institute for Health and Welfare, Finland) cell lines. Most of the viruses were analyzed using a standard plaque neutralization assay, ${ }^{24}$ whereas viruses that did not form clear plaques were analyzed using a microneutralization assay (CAV5, CAV6, CAV10, CAV16, EV71, EV78, and EV94). The neutralization assay was considered positive when the serum diluted at 1:4 reduced the number of plaques by more than $75 \%$ (plaque assay) or inhibited the ability of the virus to kill cells (microneutralization assay). The serum/plasma samples analyzed were taken between 1 and 5 years of age. The sample volume was adequate for the analysis of neutralizing antibodies against all 41 serotypes in 183 children. In 83 children, the volume was adequate for the analysis of 39-40 serotypes and in nine children for 4-21 serotypes.

\subsection{Statistical methods}

The statistical analyses were performed using $\mathrm{R}$ version 4.0.2 (2020-06-22; The R Foundation for Statistical Computing, https://www.R-project.org). Data are presented as medians, inter-quartile ranges (IQRs), and ranges, as the continuous variables were not normally distributed. The Wilcoxon test was used to assess differences in continuous variables. To determine the risk effect of different exposures, conditional logistic regression models were applied. The results are presented as odds ratios (OR) and 95\% confidence intervals (CI). $p$ Value was considered significant when $p<.05$.

\section{3 | RESULTS}

\section{1 | Prevalence of neutralizing antibodies}

The median age for the EV antibody analysis was 2.0 years, and the median age difference between case and control children was 7 days. All children were seropositive for at least one EV serotype. The median number of positive serotypes was eight (range 1-21). Most common serotypes were Echo33 (antibody prevalence 77\%), Echo30 (74\%), EV74 (58\%), CAV10 (56\%), and CVB1 (52\%).

\section{2 | Background factors}

None of the background factors differed statistically significantly between the case and control children (Table 1).

\subsection{Exposure to individual ev serotypes and risk of atopy}

Seropositivity for individual EV serotypes is shown in Table 2. Antibodies against Echo3 and Echo14 were less prevalent among 


\begin{tabular}{|c|c|c|c|}
\hline & Cases & Controls & \\
\hline & $n=138$ & $n=138$ & OR (95\% Cl), $p$ Value \\
\hline Older siblings (yes) & $77(56 \%)$ & $78(57 \%)$ & $\begin{array}{l}\text { OR } 0.97(0.59-1.6) \\
\quad p=0.898\end{array}$ \\
\hline Indoor pets (yes) & 38 (29\%) & $52(39 \%)$ & $\begin{array}{l}\text { OR } 0.7 \text { (0.43-1.13), } \\
\quad p=0.148\end{array}$ \\
\hline $\begin{array}{l}\text { Entry in day care (months, median } \\
\text { (range)) }\end{array}$ & $24(9-87)$ & $24(9-97)$ & $\begin{array}{l}\text { OR } 1.0(0.99-1.12) \\
\quad p=0.424\end{array}$ \\
\hline \multicolumn{4}{|l|}{ Paternal education } \\
\hline Elementary/lower secondary & $65(48 \%)$ & $53(39 \%)$ & ref \\
\hline $\begin{array}{l}\text { Matriculation examination/higher } \\
\text { secondary }\end{array}$ & 71 (52\%) & $84(61 \%)$ & $\begin{array}{l}\text { OR } 0.71 \text { (0.45-1.14) } \\
\quad p=0.159\end{array}$ \\
\hline \multicolumn{4}{|l|}{ Maternal education } \\
\hline Elementary/lower secondary & 42 (31\%) & $31(22 \%)$ & ref \\
\hline $\begin{array}{l}\text { Matriculation examination/higher } \\
\text { secondary }\end{array}$ & 95 (69\%) & 107 (78\%) & $\begin{array}{l}\text { OR } 0.66(0.39-1.11) \\
\quad p=0.118\end{array}$ \\
\hline \multicolumn{4}{|l|}{ Parental atopy } \\
\hline Neither & $32(27 \%)$ & $31(26 \%)$ & ref \\
\hline Either or both & $86(73 \%)$ & $86(74 \%)$ & OR 1 (0.53-1.86), $p=1$ \\
\hline \multicolumn{4}{|l|}{ Breastfeeding } \\
\hline Total (months, median (range)) & $7(0-25)$ & $8(0-24)$ & $\begin{array}{l}\text { OR } 0.99 \text { (0.94-1.05) } \\
\quad p=0.758\end{array}$ \\
\hline $\begin{array}{l}\text { Exclusive breastfeeding (months, } \\
\text { median (range)) }\end{array}$ & $1.2(0-5.5)$ & $1.3(0-6)$ & $\begin{array}{l}\text { OR } 1.01 \text { (0.86-1.19) } \\
\quad p=0.889\end{array}$ \\
\hline \multicolumn{4}{|l|}{ HLA risk for type 1 diabetes } \\
\hline Moderate risk & 94 (68\%) & 101 (73\%) & ref \\
\hline High risk & 44 (32\%) & 37 (27\%) & $\begin{array}{l}\text { OR } 1.37(0.76-2.47) \\
\quad p=0.299\end{array}$ \\
\hline Islet autoantibody positivity & $28(20 \%)$ & $27(20 \%)$ & $\begin{array}{l}\text { OR } 1.05(0.58-1.9) \\
\quad p=0.879\end{array}$ \\
\hline
\end{tabular}

TABLE 1 Background data of case children with allergic sensitization and controls case children as compared to control children (OR $0.11,95 \% \mathrm{Cl}$ 0.01-0.88, $p=.037$; and OR 0.17, 95\% Cl 0.04-0.75, $p=.019$, respectively). EV74 antibodies were more prevalent among case children (OR 1.68, 95\% Cl 1.02-2.76, $p=.04$ ). In a subgroup analysis of cases with higher likelihood of clinical symptoms ( $\mathrm{s}$-IgE-levels over $0.7 \mathrm{kU} / \mathrm{L}$ ), CAV6 was a strong protective element for atopic sensitization (OR 0.22, 95\% Cl 0.08-0.66, $p=.007$ ), while EV74 continued to show a risk association (OR 1.82, 95\% Cl 1.01-3.29, $p=.047$ ).

When case children with an allergic disease were compared with their matched controls, CAV6, CAV16, and Echo14 seropositivities were significantly less frequent in cases (OR $0.23,95 \% \mathrm{Cl} 0.07-0.81$,

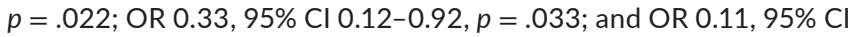
$0.01,0.88, p=.037$, respectively).

The prevalence of EV seropositivity was analyzed separately in case boys vs. control boys and case girls vs. control girls. Two EV serotypes showed significant inverse association with atopic sensitization among boys: CAV6 (OR 0.27, 95\% Cl 0.09-0.80, $p=.019$ ) and Echo14 (OR 0.10, 95\% Cl 0.01-0.78, $p=.028$ ). In addition, CAV6, CAV16, and Echo5 showed significant inverse association with allergic disease among case boys compared with their controls (OR 0.18 , $95 \% \mathrm{Cl} 0.04-0.82, p=.027$; OR 0.21, 95\% Cl 0.06-0.75, $p=.015$; and OR 0.31, 95\% Cl 0.1-0.94, $p=.039$, respectively). None of the serotypes showed association with atopic sensitization or allergic disease among girls.

\subsection{Cumulative enterovirus exposure}

The cumulative exposure to different EV serotypes illustrates the total number of different EVs that the child has encountered. We hypothesized that cumulative EV exposure would protect from allergic sensitization but found no such association (Figure 1). However, there was a trend of an inverse association between the cumulative number of EV infections and allergic disease (Figure 1). This association was statistically significant between the cumulative exposure to group A EVs and cases with atopic dermatitis (OR 0.6, 95\% Cl 0.36-0.99, $p=.048$ ).

\section{DISCUSSION}

For the first time, antibodies against a vast panel of different EVs were analyzed to shed light on the possible role of EVs as 
TABLE 2 Prevalence of neutralizing antibodies against enterovirus serotypes in cases and controls and the risk for allergic sensitization associated with enterovirus serotypes

\begin{tabular}{|c|c|c|c|c|}
\hline & $\begin{array}{l}\text { Prevalence } \\
\text { cases (\%) }\end{array}$ & $\begin{array}{l}\text { Prevalence } \\
\text { controls (\%) }\end{array}$ & OR $(95 \% \mathrm{Cl})$ & $p$ Value \\
\hline \multicolumn{5}{|c|}{ EV-A species } \\
\hline CAV4 & 25.8 & 26.7 & $0.95(0.52-1.74)$ & 0.879 \\
\hline CAV5 & 14.3 & 11.3 & $1.36(0.63-2.97)$ & 0.435 \\
\hline CAV6 & 10.1 & 18.4 & $0.48(0.22-1.01)$ & 0.053 \\
\hline CAV10 & 59.4 & 53.4 & $1.23(0.73-2.06)$ & 0.432 \\
\hline CAV16 & 12.8 & 17.3 & $0.68(0.35-1.31)$ & 0.253 \\
\hline EV71 & 7.5 & 7.5 & $1(0.38-2.66)$ & 1 \\
\hline \multicolumn{5}{|c|}{ EV-B species } \\
\hline CAV9 & 7.5 & 7.4 & $1.12(0.43-2.92)$ & 0.808 \\
\hline CVB1 & 51.4 & 52.2 & $0.97(0.59-1.59)$ & 0.9 \\
\hline CVB2 & 43.0 & 47.8 & $0.79(0.48-1.32)$ & 0.371 \\
\hline CVB3 & 10.1 & 9.4 & $1.08(0.51-2.29)$ & 0.847 \\
\hline CVB4 & 6.6 & 11.8 & $0.53(0.23-1.26)$ & 0.151 \\
\hline CVB5 & 6.6 & 5.1 & $1.14(0.41-3.15)$ & 0.796 \\
\hline CVB6 & 31.9 & 27.9 & $1.15(0.68-1.95)$ & 0.593 \\
\hline Echo1 & 27.3 & 25.9 & $1.04(0.59-1.85)$ & 0.884 \\
\hline Echo2 & 12.0 & 7.5 & $1.5(0.61-3.67)$ & 0.374 \\
\hline Echo3 & 0.8 & 6.8 & $0.11(0.01-0.88)$ & 0.037 \\
\hline Echo4 & 0.0 & 0.8 & 0 (0-Infinite) & 0.997 \\
\hline Echo5 & 35.3 & 39.1 & $0.86(0.51-1.47)$ & 0.587 \\
\hline Echo6 & 6.8 & 6.7 & $1(0.4-2.52)$ & 1 \\
\hline Echo7 & 23.3 & 14.8 & $2(0.97-4.12)$ & 0.061 \\
\hline Echo9 & 6.8 & 9.6 & $0.78(0.29-2.09)$ & 0.618 \\
\hline Echo11 & 37.5 & 38.1 & $1.04(0.6-1.8)$ & 0.889 \\
\hline Echo12 & 33.8 & 31.9 & $1.12(0.66-1.89)$ & 0.686 \\
\hline Echo13 & 5.3 & 1.5 & $3.5(0.73-16.85)$ & 0.118 \\
\hline Echo14 & 1.5 & 9.0 & $0.17(0.04-0.75)$ & 0.019 \\
\hline Echo15 & 10.5 & 14.1 & $0.71(0.34-1.48)$ & 0.356 \\
\hline Echo17 & 3.8 & 9.0 & $0.4(0.13-1.28)$ & 0.121 \\
\hline Echo18 & 4.5 & 3.7 & $1.5(0.42-5.32)$ & 0.53 \\
\hline Echo19 & 7.8 & 11.0 & $0.82(0.34-1.97)$ & 0.655 \\
\hline Echo20 & 6.0 & 3.7 & $1.6(0.52-4.89)$ & 0.41 \\
\hline Echo21 & 33.1 & 32.6 & $1.04(0.61-1.78)$ & 0.891 \\
\hline Echo25 & 3.8 & 5.2 & $0.71(0.23-2.25)$ & 0.566 \\
\hline Echo26 & 1.5 & 4.4 & $0.33(0.07-1.65)$ & 0.178 \\
\hline Echo27 & 4.5 & 5.3 & $0.83(0.25-2.73)$ & 0.763 \\
\hline Echo29 & 5.3 & 8.3 & $0.64(0.25-1.64)$ & 0.35 \\
\hline Echo30 & 75.0 & 73.3 & $1.17(0.68-2.01)$ & 0.579 \\
\hline Echo32 & 43.6 & 41.4 & $1(0.59-1.69)$ & 1 \\
\hline Echo33 & 72.9 & 81.2 & $0.62(0.35-1.12)$ & 0.112 \\
\hline EV74 & 64.7 & 51.1 & $1.68(1.02-2.76)$ & 0.04 \\
\hline EV78 & 2.3 & 3.0 & $0.5(0.09-2.73)$ & 0.423 \\
\hline \multicolumn{5}{|c|}{ EV-D species } \\
\hline EV94 & 4.5 & 4.5 & $1.25(0.34-4.66)$ & 0.739 \\
\hline
\end{tabular}

Note: The statistically significant $p$-values are presented in bold $(p<.05)$. 


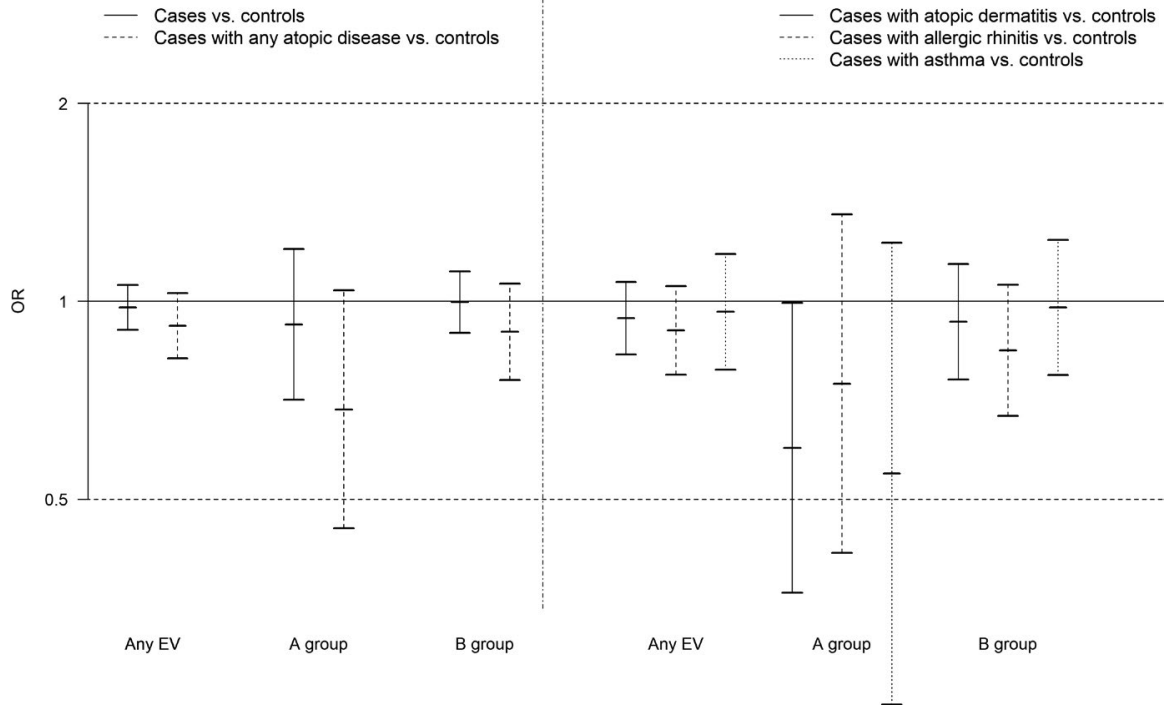

FIGURE 1 Association between cumulative enterovirus exposure for any enterovirus (EV) serotype/species $A$ serotype (A group)/species B serotype (B group), and allergic sensitization or allergic disease in cases vs. controls (left) and for cases with a specific allergic disease versus their matched controls (right) risk-modifying factors in the development of allergic sensitization and/or disease. The cumulative number of infections by different EV serotypes was not associated with IgE sensitization. However, the cumulative number of species A EV infections showed a trend for protective association with the development of allergic disease. This inverse association was significant for IgE-sensitized cases with atopic dermatitis, whereas for hay fever and asthma, only a statistically insignificant inverse tendency was observed. In particular, asthma is a heterogeneous disease and many patients lack atopic background, ${ }^{25}$ which could explain the lack of association. Another explanation could be limited statistical power, since a number of cases with asthma or hay fever were lower than those with atopic dermatitis.

Atopic sensitization is often asymptomatic with no impact on quality of life or health costs. By contrast, the burden of atopic diseases on both the patient and the society can be significant. From this point of view, means to prevent atopic diseases would have a greater impact than means to prevent atopic sensitization. Our results signal that the risk-modifying factors associated with atopic sensitization and disease may differ. Thus, they should be viewed separately in future studies to find ways to protect from the actual clinical disease. In addition, it is possible that the risk-modifying factors can differ between the various forms of atopic diseases, as suggested in our study.

Certain individual EV serotypes showed an association with IgE sensitization and/or allergic disease, including Echo3 and Echo14, which both showed an inverse association, and EV74, which showed a risk association. Interestingly, Echo3 has been reported to associate with a reduced risk of allergic diseases also in a previous study ${ }^{11}$ Additionally, we wish to highlight CAV6, CAV16, Echo5, and Echo14 as intriguing protective players in the development of allergic diseases. In particular, CAV6 exposure, a common virus causing hand, foot, and mouth disease, was significantly less frequent in cases with allergic disease than in their matched controls and CAV6 was also less frequent in cases with stronger ( $>0.7 \mathrm{kU} / \mathrm{L}$ ) IgE sensitization. The inverse CAV6 association was observed for boys with IgE sensitization and boys with atopic disease, but not for girls. This sex difference is in parallel with our previous report of rhinoviruses' association with reduced risk of atopy within boys but not in girls ${ }^{21}$ and indicates that viruses' influence on atopy risk may be more pronounced in boys. The fact that only a few of the $41 \mathrm{EV}$ serotypes showed association with atopy may relate to immunophenotype differences between virus serotypes and strains, as suggested by our previous report showing very different immune system activation by individual CBV1 strains. ${ }^{26}$ It is also known that EVs differ in their ability to infect various white blood cell populations. ${ }^{27}$ Thus, it is possible that certain EV types, such as CAV6, have a unique "anti-allergic" immunophenotype due to their specific interaction with the immune system. This hypothesis requires further studies.

Previous studies have not identified any specific microbes driving the inverse association between microbial exposures and the risk of allergic disease. Instead, it seems that a vast variety of microbes, perhaps even the total microbiome, is involved. ${ }^{4}$ Our finding of an inverse association between the cumulative exposure to different EV types and allergic disease fits with the hypothesis of more than a single culprit microbe involved. Accordingly, even though we found some significant associations when analyzing the individual serotypes, we want to encourage opting for as large virus panel as possible in future studies on EVs and atopy.

The strengths of this study are the comprehensive panel of the most common circulating EV serotypes and the utilization of a highly serotype-specific neutralization assay, which can be considered the gold standard for determining serotype-specific EV antibodies. The endpoints of our study were also solid, as the IgE sensitization was determined with a wide allergen panel including both inhalant and food allergens, and information on allergic diseases was based on either a validated atopy questionnaire (ISAAC) or a diagnosis made by a physician.

The limitations of the study include the lack of correction of $p$ values for the great number of comparisons performed when analyzing the individual EV serotypes. In our study, using a Bonferroni 
coefficient of 41 (number of EV serotypes studied) leads to a statistical significance cutoff value of $p<.0012$ and wipes out the significant associations for individual serotypes. Bonferroni correction has been criticized for being overly conservative, and therefore, we held to the generally accepted $p$ value $<.05$. One must keep in mind that without correction for multiple comparisons, the results for individual EV serotypes must be kept more hypothesis-generating than proving evidence. Though the findings for individual serotypes are preliminary, they suggest a biologically plausible hypothesis of certain EV serotypes having a riskmodifying effect on atopy. This will hopefully inspire additional studies to confirm and further clarify the influence of these serotypes on the risk of atopy.

A further limitation is that the study subjects did not represent the general population but carried specific type 1 diabetesassociated HLA haplotypes. However, no consistent relationship has been shown between allergic diseases and type 1 diabetes, ${ }^{28,29}$ and neither islet autoimmunity nor HLA was associated with atopic sensitization in the current cohort. Lastly, we were unable to ascertain the chronological order of the EV infections and IgE sensitization, as the latter was measured only at the age of 5 years.

In conclusion, we show that there is an inverse association between cumulative EV infections and allergic disease but not with allergic sensitization alone. Further studies are needed to consolidate this finding and to evaluate whether it involves causality and obstruction of disease progression from sensitization to allergic disease by EVs. We also want to highlight serotypes Echo3, Echo5, Echo14, CAV6, and CAV16 as possible protective serotypes and EV74 as a possible risk factor for allergic sensitization and disease.

\section{ACKNOWLEDGEMENTS}

The authors would like to thank the families who have participated in the DIPP study, as well as the study nurses and other personnel running the study.

\section{CONFLICT OF INTEREST}

The authors declare no conflicts of interest.

\section{AUTHOR CONTRIBUTIONS}

Tiina Palmu: Conceptualization (equal); Formal analysis (supporting); Visualization (equal); Writing-original draft (lead). Jussi Lehtonen: Formal analysis (lead); Visualization (equal); Writing-review \& editing (equal). Laura Korhonen: Resources (supporting); Writing-review \& editing (equal). Suvi M. Virtanen: Investigation (equal); Writingreview \& editing (equal). Onni Niemelä: Investigation (equal); Writing-review \& editing (equal). Jorma Toppari: Project administration (equal); Writing-review \& editing (equal). Jorma llonen: Project administration (equal); Writing-review \& editing (equal). Riitta Veijola: Project administration (equal); Writing-review \& editing (equal). Mikael Knip: Project administration (equal); Writing-review \& editing (equal). Olli H. Laitinen: Resources (supporting); Writingreview \& editing (equal). Maria Lönnrot: Conceptualization (equal); Writing-review \& editing (equal). Heikki Hyöty: Conceptualization (equal); Project administration (equal); Resources (lead); Writingreview \& editing (equal).

\section{PEER REVIEW}

The peer review history for this article is available at https://publo ns.com/publon/10.1111/pai.13577.

\section{ORCID}

Tiina Palmu (D) https://orcid.org/0000-0002-7191-7938

Suvi M. Virtanen (D) https://orcid.org/0000-0001-8928-0878

Mikael Knip (D) https://orcid.org/0000-0003-0474-0033

\section{REFERENCES}

1. Kulig M, Bergmann R, Klettke U, Wahn V, Tacke U, Wahn U. Natural course of sensitization to food and inhalant allergens during the first 6 years of life. J Allergy Clin Immunol. 1999;103(6):1173-1179.

2. Burney P, Malmberg E, Chinn S, Jarvis D, Luczynska C, Lai E. The distribution of total and specific serum IgE in the European community respiratory health survey. I Allergy Clin Immunol. 1997;99(3):314-322.

3. Law M, Morris JK, Wald N, Luczynska C, Burney P. Changes in atopy over a quarter of a century, based on cross sectional data at three time periods. BMJ. 2005;330(7501):1187-1188.

4. Liu AH. Revisiting the hygiene hypothesis for allergy and asthma. $J$ Allergy Clin Immunol. 2015;136(4):860-865.

5. Haahtela T.A biodiversity hypothesis.Allergy. 2019;74(8):1445-1456

6. Lehar SM, Bevan MJ. Immunology: polarizing a T-cell response. Nature. 2004;430(6996):150-151.

7. Mustonen K, Orivuori L, Keski-Nisula L, et al. Inflammatory response and IgE sensitization at early age. Pediatr Allergy Immunol. 2013;24(4):395-401.

8. Linneberg A, Ostergaard C, Tvede M, et al. IgG antibodies against microorganisms and atopic disease in Danish adults: the Copenhagen allergy study. J Allergy Clin Immunol. 2003;111(4):847-853.

9. Matricardi PM, Rosmini F, Riondino S, et al. Exposure to foodborne and orofecal microbes versus airborne viruses in relation to atopy and allergic asthma: epidemiological study. BMJ. 2000;320(7232):412-417.

10. Silverberg JI, Norowitz KB, Kleiman E, et al. Association between varicella zoster virus infection and atopic dermatitis in early and late childhood: a case-control study. J Allergy Clin Immunol. 2010;126(2):300-305.

11. Korhonen L, Kondrashova A, Tauriainen S, et al. Enterovirus infections in early childhood and the risk of atopic disease - a nested case-control study. Clin Exp Allergy. 2013;43(6):625-632.

12. Seiskari T, Kondrashova A, Tauriainen S, et al. Role of enterovirus infections in IgE sensitization. J Med Virol. 2012;84(2):268-271.

13. Nilsson C, Linde A, Montgomery SM, et al. Does early EBV infection protect against IgE sensitization? J Allergy Clin Immunol. 2005;116(2):438-444.

14. Reimerink J, Stelma F, Rockx B, et al. Early-life rotavirus and norovirus infections in relation to development of atopic manifestation in infants. Clin Exp Allergy. 2009;39(2):254-260.

15. Janse JJ, Wong GWK, Potts J, et al. The association between foodborne and orofecal pathogens and allergic sensitisation EuroPrevall study. Pediatr Allergy Immunol. 2014;25(3):250-256.

16. de Crom SCM, Rossen JWA, van Furth AM, Obihara CC. Enterovirus and parechovirus infection in children: a brief overview. Eur J Pediatr. 2016;175(8):1023-1029.

17. Drummond CG, Bolock AM, Ma C, Luke CJ, Good M, Coyne $C B$. Enteroviruses infect human enteroids and induce antiviral 
signaling in a cell lineage-specific manner. Proc Natl Acad Sci USA. 2017;114(7):1672-1677.

18. Vreugdenhil GR, Wijnands PGJTB, Netea MG, van der Meer JWM, Melchers WJG, Galama JMD. Enterovirus-induced production of pro-inflammatory and T-helper cytokines by human leukocytes. Cytokine. 2000;12(12):1793-1796.

19. Korhonen L, Seiskari T, Lehtonen J, et al. Enterovirus infection during pregnancy is inversely associated with atopic disease in the offspring. Clin Exp Allergy. 2018;48(12):1698-1704.

20. Lee Z-M, Huang Y-H, Ho S-C, Kuo H-C. Correlation of symptomatic enterovirus infection and later risk of allergic diseases via a population-based cohort study. Medicine (Baltimore). 2017;96(4):e5827.

21. Korhonen L, Oikarinen S, Lehtonen J, et al. Rhinoviruses in infancy and risk of immunoglobulin E sensitization. J Med Virol. 2019;91(8):1470-1478.

22. Kupila A, Muona P, Simell T, et al. Feasibility of genetic and immunological prediction of type I diabetes in a population-based birth cohort. Diabetologia. 2001;44(3):290-297.

23. Laitinen $\mathrm{OH}$, Honkanen $\mathrm{H}$, Pakkanen $\mathrm{O}$, et al. Coxsackievirus $\mathrm{B} 1$ is associated with induction of -cell autoimmunity that portends type 1 diabetes. Diabetes. 2014;63(2):446-455.

24. Roivainen $M$, Knip M, Hyöty $H$, et al. Several different enterovirus serotypes can be associated with prediabetic autoimmune episodes and onset of overt IDDM. Childhood Diabetes in Finland (DiMe) Study Group. J Med Virol. 1998;56(1):74-78.

25. Christiansen ES, Kjaer HF, Eller E, et al. The prevalence of atopic diseases and the patterns of sensitization in adolescence. Pediatr Allergy Immunol. 2016;27(8):847-853.

26. Hämäläinen $\mathrm{S}$, Nurminen $\mathrm{N}$, Ahlfors $\mathrm{H}$, et al. Coxsackievirus $\mathrm{B} 1$ reveals strain specific differences in plasmacytoid dendritic cell mediated immunogenicity. J Med Virol. 2014;86(8):1412-1420.

27. Vuorinen T, Vainionpää R, Heino J, Hyypiä T. Enterovirus receptors and virus replication in human leukocytes. J Gen Virol. 1999;80(4):921-927.

28. Seiskari T, Viskari H, Kondrashova A, et al. Co-occurrence of allergic sensitization and type 1 diabetes. Ann Med. 2010;42(5):352-359.

29. Strömberg LG, Ludvigsson GJ, Björkstén B. Atopic allergy and delayed hypersensitivity in children with diabetes. J Allergy Clin Immunol. 1995;96(2):188-192.

How to cite this article: Palmu T, Lehtonen J, Korhonen L, et al. Association of different enteroviruses with atopy and allergic diseases in early childhood. Pediatr Allergy Immunol. 2021;32:1629-1636. https://doi.org/10.1111/pai.13577 